\title{
Study into COVID-19 Crisis Using Primary Care Mental Health Consultations and Prescriptions Data
}

\author{
Agnieszka LEMANSKA ${ }^{\mathrm{a}, \mathrm{b}, 1}, \mathrm{Uy} \mathrm{HOANG}^{\mathrm{c}}$, Nathan JEFFREYS ${ }^{\mathrm{d}}$, Clare BANKHEAD $^{\mathrm{c}}$, \\ Kam BHUI $^{\mathrm{c}, \mathrm{e}}$, Filipa FERREIRA ${ }^{\mathrm{c}}$, Sally HARCOURT ${ }^{\mathrm{f}}$, Anthony JAMES ${ }^{\mathrm{e}}$, Harshana \\ LIYANAGE $^{c}$, Brian D NICHOLSON ${ }^{c}$, Julian SHERLOCK ${ }^{\mathrm{c}}$, Gillian SMITH ${ }^{\mathrm{f}}$, Nadia A. \\ S. SMITH ${ }^{\text {a }}$, Spencer A.THOMAS ${ }^{\text {, }}$, John WILLIAMS ${ }^{\mathrm{c}}$ and Simon De LUSIGNAN ${ }^{\mathrm{c}}$ \\ a Data Science Department, National Physical Laboratory, Teddington, UK \\ ${ }^{\mathrm{b}}$ School of Health Sciences, University of Surrey, Guildford, UK \\ ${ }^{\mathrm{c}}$ Nuffield Department of Primary Care Health Sciences, University of Oxford, UK \\ ${ }^{\mathrm{d}}$ Royal Surrey NHS Foundation Trust, Guildford, UK \\ ${ }^{\mathrm{e}}$ Department of Psychiatry, University of Oxford, UK \\ ${ }^{\mathrm{f}}$ National Infection Service, Public Health England, Birmingham, UK
}

\begin{abstract}
The effect of the 2020 pandemic, and of the national measures introduced to control it, is not yet fully understood. The aim of this study was to investigate how different types of primary care data can help quantify the effect of the coronavirus disease (COVID-19) crisis on mental health. A retrospective cohort study investigated changes in weekly counts of mental health consultations and prescriptions. The data were extracted from one the UK's largest primary care databases between January 1st 2015 and October 31st 2020 (end of follow-up). The 2020 trends were compared to the 2015-19 average with $95 \%$ confidence intervals using longitudinal plots and analysis of covariance (ANCOVA). A total number of 504 practices $(7,057,447$ patients) contributed data. During the period of national restrictions, on average, there were $31 \%(3957 \pm 269, \mathrm{p}<0.001)$ fewer events and $6 \%(4878 \pm 1108, \mathrm{p}<0.001)$ more prescriptions per week as compared to the 2015 19 average. The number of events was recovering, increasing by $75( \pm 29, p=0.012)$ per week. Prescriptions returned to the 2015-19 levels by the end of the study ( $p=$ 0.854). The significant reduction in the number of consultations represents part of the crisis. Future service planning and quality improvements are needed to reduce the negative effect on health and healthcare.
\end{abstract}

Keywords. Mental health, COVID-19, pandemic, lockdown, real-world evidence

\section{Introduction}

The COVID-19 pandemic, and the national and worldwide efforts to contain and manage the infections, have challenged all facets of life and placed an unprecedented strain on people, patients and healthcare systems. Continued social distancing and isolation measures, coupled with limited access to healthcare and face-to-face consultations are challenging for everyone, but people with mental health conditions are especially

1 Corresponding Author, Dr Agnieszka Lemanska, Data Science, National Physical Laboratory, Teddington, United Kingdom; E-mail: agnieszka.lemanska@npl.co.uk 
vulnerable. In mid-March 2020 (week 11), the UK government started introducing national restrictions. The aim was to control the rate of infections, prevent an overburden on the National Health Service (NHS) and reduce the number of deaths associated with the COVID-19. However, the emerging research indicates that the pandemic and the lockdown affected mental health of the population. For example, increased anxiety and depression in UK adults experiencing isolation have been reported [1]. The effect of the 2020 pandemic and the measures introduced to control it is not yet fully understood. In this work, the 2020 trends in primary care events (consultations) and prescriptions data for mental health conditions such as anxiety, depression, and sleep disorders were compared to 2015-19 average to understand the effect of the pandemic and lockdown on mental health and on access to healthcare. This is important because as restrictions ease, it is necessary to plan future workloads and consider how to improve healthcare services provided to people in the UK and worldwide during pandemics. This work has applications beyond mental health, as the emerging research indicates that both mental and physical health are affected, for example by delayed diagnosis of long term conditions such as cancer or diabetes [2].

\section{Methods}

\subsection{Study design, data extraction}

This was a population-based, longitudinal retrospective cohort study using the Oxford Royal College of General Practitioners (RCGP) Clinical Informatics Digital Hub (ORCHID) database. ORCHID extracts electronic healthcare records (EHRs) from one of the UK's biggest primary care sentinel networks. ORCHID is representative of the English population[3] and comprises $>500$ practices with nearly 10 million patient records ( $>10 \%$ of the English population). Two types of data were used: 1) events (primarily consultations) and 2) prescriptions data. Events were extracted from EHRs using clinical codes in the SNOMED-CT system. They included consultations, diagnosis and symptoms for common mental health conditions such as anxiety, depression and sleep disorders. The prescriptions data were extracted with lists of medications for pharmacological groups such as antidepressants, anxiolytics and hypnotics (sleep medications). These lists were generated based on the British National Formulary (BNF78, 2019-2020, bnf.org). The lists of SNOMED-CT codes and medications can be obtained from the corresponding author. The study was from January $1^{\text {st }} 2015$ to October $31^{\text {st }} 2020$, when the follow-up ended.

\subsection{Statistical analysis}

Weekly counts of events and prescriptions were calculated and plotted for the year 2020 against the 2015 to 2019 average with $95 \%$ confidence intervals. Analysis of covariance (ANCOVA) was used to estimate the difference in the weekly counts between 2020 and the 2015-19 average for the period of week 12 (beginning of lockdown) to week 43 (end of follow-up) and its statistical significance adjusted for the weekly trend. The binary grouping variable for ANCOVA was year 2020 vs year 2015-19 average. The week variable was a covariate. Database extraction was in Structured Query Language (SQL) Server Management Studio version 18.3.1 and statistical analyses in R version 3.5.1. 


\subsection{Approvals}

This study was approved by the RCGP board (data request number RSC_1620). Ethical approval was not required. Patients consented to the research on an opt-out basis.

\section{Results}

\subsection{The cohort, events and prescription counts}

In total, 504 practices and 7,057,447 participants contributed data (over 5 million participants each year due to registering and de-registering). There were over 5 million events recorded for $1,205,825(17 \%)$ participants and nearly 26 million prescriptions for $1,459,223(21 \%)$ participants. Table 1 presents the yearly split and rates per 100,000 patients.

Table 1. Weekly counts of events (primarily consultations) and prescriptions

\begin{tabular}{|c|c|c|c|c|c|}
\hline & Participants & Events & $\begin{array}{l}\text { Rate per } \\
100,000\end{array}$ & Prescriptions & $\begin{array}{l}\text { Rate per } \\
100,000\end{array}$ \\
\hline Total, 2015- 2020 & $7,057,447$ & $5,000,003$ & & $25,755,647$ & \\
\hline 2015 & $5,080,326$ & $877,569(18 \%)$ & 17,274 & $4,142,322(16 \%)$ & 81,537 \\
\hline 2016 & $5,112,815$ & $888,096(18 \%)$ & 17,370 & $4,323,328(17 \%)$ & 84,559 \\
\hline 2017 & $5,173,919$ & $883,931(18 \%)$ & 17,084 & $4,390,547(17 \%)$ & 84,859 \\
\hline 2018 & $5,250,640$ & $864,950(17 \%)$ & 16,473 & 4,414,077 (17\%) & 84,067 \\
\hline 2019 & $5,345,376$ & $862,485(17 \%)$ & 16,135 & $4,526,997(18 \%)$ & 84,690 \\
\hline 2020 (Jan - Oct $)$ & $5,202,588$ & $622,972(12 \%)$ & & $3,958,376(15 \%)$ & \\
\hline Whole 2020 (estimate) & & $747,566(15 \%)$ & 14,369 & $4,750,051(18 \%)$ & 91,301 \\
\hline
\end{tabular}

Trends in 2020 compared to previous years (2015 to 2019)

From week 11 onwards, when the national restrictions started, the 2020 count of events dropped sharply below the 2015-19 average (Figure 1A). Although the numbers were gradually recovering, events remained below until the end of the study. The weekly count of prescriptions (Figure 1B) followed a different trend. It was above the 2015-19 average before the lockdown. We observed a peak increase in week 12 when the lockdown started and then the numbers returned to the 2015-19 values.
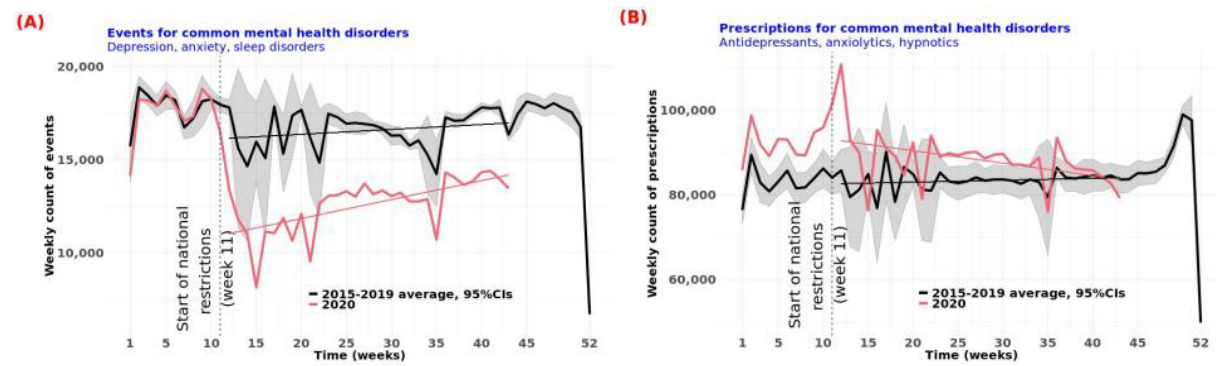

Figure 1. Trends in weekly counts of (A) events and (B) medications for common mental health disorders 


\subsection{The ANCOVA analysis}

In both ANCOVA models the grouping variable (Year), the covariate (Week), and the interaction term were determined to be significant (Table 2). We therefore report that in the period of week 12 to 43 of 2020 there were on average 12,599 events per week which was $31 \%(3,957 \pm 269, \mathrm{p}<0.001)$ less than the $2015-19$ average for the same period. The 2020 weekly counts were slowly recovering towards the 2015-19 average, increasing by around $1 \%(75 \pm 29, \mathrm{p}=0.012)$ events each week. Prescriptions followed a different trend. There were on average $6 \%(4,878 \pm 1108, \mathrm{p}<0.001)$ more prescriptions per week in weeks 12 to 43 of 2020 than 2015-19. The 2020 prescription counts returned to the 2015-19 levels with no statistically significant difference in week $43(\mathrm{p}=0.854)$.

Immediately following the start of lockdown (week 12), a significant drop by $46 \%$ $(5,123 \pm 526, \mathrm{p}<0.001)$ in consultations was observed and a peak increase in prescriptions with $11 \%(10,154 \pm 2,164, \mathrm{p}<0.001)$ more prescriptions issued that week than the 2015-19 average.

Table 2. ANCOVA analysis of weekly counts of (A) events and (B) prescriptions

\begin{tabular}{llccc} 
(A) Events & Covariate value & Coefficient & Standard Error & p-value \\
\hline Year 2020 & Week 12 (start) & 11,024 & 372 & $<0.001$ \\
2015-19 average & & 5,123 & 526 & $<0.001$ \\
Year 2020 & Week 27.5 (mean) & 12,599 & 190 & $<0.001$ \\
2015-19 average & & 3,957 & 269 & $<0.001$ \\
Year 2020 & Week 43 (end of & 14173 & 372 & $<0.001$ \\
2015-19 average & follow-up) & 2,790 & 526 & $<0.001$ \\
Week & & 102 & 21 & $<0.001$ \\
Week * 2015-19 average & & -75 & 29 & 0.012 \\
\hline (B) Prescriptions & Covariate value & Coefficient & Standard Error & p-value \\
\hline Year 2020 & Week 12 (start) & 92,784 & 1530 & $<0.001$ \\
$2015-19$ average & & $-10,154$ & 2,164 & $<0.001$ \\
Year 2020 & Week 27.5 (mean) & 88,208 & 783 & $<0.001$ \\
$2015-19$ average & & $-4,878$ & 1108 & $<0.001$ \\
Year 2020 & Week 43 (end of & 83,633 & 1530 & $<0.001$ \\
$2015-19$ average & follow-up) & 399 & 2,164 & 0.854 \\
Week & & -295 & 85 & $<0.001$ \\
Week $* 2015-19$ average & & 340 & 120 & 0.006 \\
\hline
\end{tabular}

\section{Discussion}

By using two different types of data, we were able to investigate the impact of the coronavirus crisis on primary care. A significant drop in consultations was observed following lockdown. Prescriptions followed an opposite trend with more prescriptions issued during the national restrictions period than in the years from 2015 to 2019 . These finding and the learning form this project is important. This is because as we recover from the current pandemic, we need to review strengths and weaknesses of the healthcare services, and we need to put crisis-related plans in place for future national and global emergencies.

This research adds to the emerging evidence that the COVID-19 pandemic, and the measures to control it, may have indirectly affected mental health[2] as well as physical health $[2,4,5]$ of the population and the quality, quantity and type of services available to people. Although a drop in consultations might be expected in a lockdown when 
people are self-isolating and shielding, but potentially they may also neglect to seek help. The increase in prescriptions indicates the reliance on medications (potentially without sufficient follow-ups), rather than considering other therapeutic options.

It is important to note that the results indicate a drop in consultations but not in rates of mental health disorders. This therefore supports the need for different mechanisms of delivering consultations, including remote appointments via phone or internet. Research is needed to investigate the effectiveness and availability of remote consultations.

\section{Conclusion}

With the use of two different types of primary care data, we were able to improve the understanding of strengths and limitations of healthcare services in the UK. This learning can help improve the resilience of healthcare systems for a future crisis.

\section{Acknowledgements}

This study was funded by the department of Business, Energy and Industrial Strategy (BEIS) through the cross-theme National Measurement Strategy under the Life Sciences \& Healthcare theme (Digital Health [122471] Data Curation) at the National Physical Laboratory (NPL), UK.

\section{References}

[1] Robb CE, et al. Associations of Social Isolation with Anxiety and Depression During the Early COVID19 Pandemic: A Survey of Older Adults in London, UK. Front Psychiatry, 2020. 11.

[2] Williams R, et al. Diagnosis of physical and mental health conditions in primary care during the COVID19 pandemic: a retrospective cohort study. The Lancet Public Health. 2020;5(10).

[3] Correa A, et al. Royal College of General Practitioners Research and Surveillance Centre (RCGP RSC) sentinel network: a cohort profile. BMJ Open. 2016;6(4).

[4] Mesnier J, et al. Hospital admissions for acute myocardial infarction before and after lockdown according to regional prevalence of COVID-19 and patient profile in France: a registry study. The Lancet Public Health. 2020;5(10).

[5] Earnshaw, $\mathrm{CH}$, et al. Reduction in skin cancer diagnosis, and overall cancer referrals, during the COVID19 pandemic. British Journal of Dermatology. 2020;183(4). 